\title{
MedienPädagogik
}

Zeitschrift für Theorie und Praxis der Medienbildung

www.medienpaed.com

ISSN 1424-3636

Jahrbuch Medienpädagogik 17:

Lernen mit und über Medien in einer digitalen Welt

Herausgegeben von Klaus Rummler, Ilka Koppel, Sandra Aßmann,

Patrick Bettinger und Karsten D. Wolf

\section{Big Data als datenbasierte Programmierung}

\section{Eine medienpädagogische Analyse künstlicher neuronaler Netzwerke}

Christian Swertz

\section{Zusammenfassung}

In medienpädagogischen Theorien wird oft auf Analysen medieninduzierter gesellschaftlicher Entwicklungen oder auf Analysen der Inhalte von Medien und ihre Struktur zurückgegriffen. Diese Perspektiven werden in der relationalen Medienpädagogik um die Analyse der Technologie von Medien ergänzt. Im Beitrag wird aus dieser Sicht eine Analyse der datenbasierten Programmierung als Technologie in der Absicht vorgenommen, die medienpädagogische Relevanz der Technologie zu untersuchen.

\section{Big Data as Data Based Coding. A Media Educational Analysis of Artificial Neuronal Networks}

\begin{abstract}
Theories in Media Education are often based on the analysis of developments in society caused by media or on the analysis of content. In the Relational Media Education Theory, these perspectives are complemented by an analysis of the
\end{abstract}


technology applied in media. Based on this perspective, data based programming techniques are analysed in order to research the relevance of the technology for media education.

\section{Einleitung}

Computertechnologie zu diskutieren ist nicht einfach, wenn Digitalisierungsprozesse sich in die «neuzeitliche Episteme» (Jörissen 2016) so eingeschrieben haben, dass sie die Selbstverhältnisse des Menschen bestimmen, und Algorithmen «viel unkontrollierbarer als alle anderen nicht menschlichen Akteure der kompletten Kulturgeschichte» (ebd.) sind. Denn dann können zwar Algorithmen den Menschen diskutieren und als Mittel für ihre Zwecke verwenden. Menschen können aber bestenfalls den Spuren von Algorithmen und Digitalisierungsprozessen nachgehen, diese aber nicht souverän reflektieren oder gestalten.

Damit wäre die medienpädagogische Aufgabe klar: Die Würde der Algorithmen ist zu vermitteln. Wenn aber der Kolonialisierung von Lebenswelt durch Digitalisierungsprozesse und Algorithmen, d.h. durch digitale Technik und Technologie, nicht von vornherein stattgegeben werden soll, muss zunächst geklärt werden, wie digitale Technik und Technologie reflektiert werden können.

Ein erster Ausgangspunkt medienpädagogischer Reflexionen sind gesellschaftliche Veränderungen. So hat z.B. Bachmair das Verständnis aktueller Entwicklungen als disparate Kultur (Bachmair 2017) und als Enttraditionalisierung, als reflexive Moderne und als Erlebnisrationalität (Bachmair 2005) zum Ausgangspunkt medienpädagogischer Überlegungen gemacht. Damit werden soziologische Beschreibungen als Grundlagen der medienpädagogischen Analyse und des medienpädagogischen Handelns verwendet. Diese soziologischen Analysen liefern relevante Beschreibungen, ermöglichen es aber nicht, Enttraditionalisierungsprozesse und ähnliche Veränderungen als Ausdruck der mit Algorithmen gesetzten Zwecke zu verstehen. Auf dieser Grundlage ist es daher nur schwer möglich, Technik und Technologie souverän zu reflektieren.

Ein zweiter Ausgangspunkt medienpädagogischer Theoriebildung sind die Inhalte von Medien. In dieser Perspektive werden Inhalte entweder 
als bedrohlich (Hemmerich 2017) oder im Blick auf ihren bildenden Gehalt (Jörissen und Marotzki 2009) analysiert. In diesen Perspektiven, die in der Medienpädagogik unter den Stichworten «Bewahrpädagogik» und «strukturale Medienbildung» diskutiert werden, kommen weder Technik noch Technologie vor, die also auch nicht reflektiert werden.

Ein dritter Ausgangspunkt ist die Analyse der medialen Infrastruktur. Dabei wurde insbesondere die digitale Technik und die damit verwendete Software zum Gegenstand von Analysen gemacht. So haben Damberger und Iske den bildenden Gehalt von kulturellen Praktiken, die als «Quantified Self» analysiert werden (Damberger und Iske 2017), herausgearbeitet und Jörrissen und Verständig eine Analyse von Code und Software aus machttheoretischer Perspektive vorgelegt (Jörissen und Verständig 2017). Während Damberger und Iske zwischen den sich bildenden Menschen und der in Bildungsprozessen verwendeten Technik unterscheiden, schreiben Jörissen und Verständig der Technik und dem Menschen die gleiche Emergenz zu (ebd.: 41). Das entspricht einem kybernetischem Ansatz, mit dem kein Unterschied zwischen Technik und Leben gemacht wird. Technik und Menschen werden als Informationsprozesse begriffen. Damit ist eine Kritik nicht möglich, weil Information nur um den Preis der Selbstzerstörung kritisiert werden kann, wenn alles Information ist.

Insofern ist es nicht überraschend, dass von Jörissen und Verständig nicht auf den angesichts des von ihnen vorgenommenen Problems naheliegenden Ansatz der Apparatustheorie (Baudry 1994) zurückgreifen. Denn mit der Apparatustheorie wird reflektiert, wie durch das Ensemble der Apparate (digitaler Raum) die dominante Ideologie den Benutzerinnen und Benutzern eingeprägt wird (Winkler 2003). Solche unbewussten Vorgänge werden von den Vertreterinnen und Vertretern der Apparatustheorie als dem Bewusstsein zugänglich und also als kritisierbar gedacht. Ähnliches gilt für Ansätze, in denen eine Analyse von Produktionsmitteln - und Daten werden mit Produktionsmitteln produziert - im Blick auf gesellschaftliche Prozesse prominent durchgeführt worden ist (Marx 1962).

Berücksichtigt wird die Möglichkeit der Kritik indes im Ansatz der metakommunikativen Medienpädagogik. So betont Baacke, dass in einem Buch über Kommunikation dieses zugleich Gegenstand der Betrachtung ist (Baacke 1973, 11). Mit der damit angezeigten selbstreflexiven Perspektive 
von Baacke als sich selbst reflektierendem Autor, die Baacke mit einem wissenschaftstheoretisch gewendeten Metakommunikationsbegriff erläutert hat (ebd., 355), kann die eigene Betroffenheit distanziert, die Beschreibung des Gegenstandes mit einer Orientierung an der souveränen Gestaltung des Gegenstandes in nicht-diskursiven Praktiken (Barberi 2018, 87) erweitert und so die Orientierung an der Würde der Algorithmen vermieden werden.

Das leistet auch die relationale Medienpädagogik von Meder, die für das hier zur Diskussion stehende Problem gegenüber der metakommunikativen Medienpädagogik den Vorteil hat, dass Meder auch eine genaue Analyse digitaler Infrastrukturen vorgenommen hat. Daher wird die relationale Medienpädagogik als Grundlage der folgenden Analyse verwendet. Das erfordert eine kurze Darstellung der Position.

In der relationalen Medienpädagogik werden wie in der metakommunikativen Medienpädagogik Reflexion (Medienkritik) und Design (Mediengestaltung) berücksichtigt. Zudem werden im Gebrauch digitaler Medien veränderte kulturelle Praktiken mit einem reformulierten Bildungsbegriff eingeholt (Meder 2004) Dabei wird der Gegenstand durch eine realdialektische Reflexion distanziert.

Ein entscheidender Punkt ist, dass aus dieser Sicht ein «Werden» nur dem Menschen zukommt, nicht aber der Technik (Meder 2016). Mit dieser Differenz können Gestaltungspotentiale aufgezeigt und Reflexion und Design von Medien (einschliesslich medienbezogener Machtprozesse) zum Inhalt medienpädagogischer Theorie und medienpädagogischer Praxis gemacht werden.

Die Berücksichtigung von Reflexion und Design bzw. von Theorie und Praxis macht es erforderlich, nicht nur Orientierungswissen, sondern auch Orientierungskönnen zu berücksichtigen. Bildung und Ausbildung oder, in der Terminologie der Frankfurter Erklärung zur Bildung in einer digitalen Welt (Weich 2019), Analyse, Reflexion und Gestaltung, sind gleichermassen zu berücksichtigen. Mit dem allgemeinen Medienbegriff der relationalen Medienpädagogik (Swertz 2009; Meder 2014) werden Medien dabei als ein Problem verstanden, das alle Bereiche der Lebenswelt betrifft. Diese Fassung des Medienbegriffs ist einerseits wie andere Ansätze durch die empirische Beobachtung motiviert, dass viele Menschen sich dafür 
entschieden haben, Medien einen zentralen Stellenwert in ihrer Kultur zu geben. Medien können daher als Kulturtechniken verstanden werden ( $\mathrm{Me}-$ der 2015). Andererseits werden Medien in einer transzendentalkritischen Analyse distanziert, indem eine "grundsätzliche Asymmetrie zwischen Was-Bewusstsein und Dass-Bewusstsein» (Meder 2016, 185) angesetzt und im Vollzug wieder eingeholt wird. Damit wird der Gegenstandsbereich der Medienpädagogik markiert und eine Medien reflexiv distanzierende Perspektive entwickelt. Medien so einer souverän und reflexiv distanzierenden Analyse zu unterwerfen, impliziert die Transformation des Problems in eine medienpädagogische Aufgabe.

Aus Sicht der relationalen Medienpädagogik ist klar, dass die Medienpädagogik als Wissenschaft nur um den Preis des Gestaltungsverzichts als diverse und poröse Disziplin (Knaus 2018) verstanden werden kann. So zurückhaltend wird in der Medienpädagogik aber kaum gearbeitet: Die Konzentration auf Orientierungskönnen wird statt der Beschränkung auf Orientierungswissen unter der Bezeichnung Handlungsorientierte Medienpädagogik seit längerem diskutiert (Hüther und Podehl 2017).

Medienpädagogik trotz der eingangs markierten Differenzen so zu begreifen stellt einen so breiten Konsens dar, dass es schwerfällt, Ansätze, in denen die Bezeichnung Medienkompetenz in den Mittelpunkt gerückt wird, von solchen abzugrenzen, in denen die Bezeichnung Medienbildung als zentral erachtet wird (Schorb 2009), oder verschiedene Medienkompetenzbegriffe zu unterscheiden (Schiefner-Rohs 2012): Über den Kern der Sache besteht Konsens.

Das gilt auch für die Berücksichtigung von Erkenntnissen anderer Disziplinen. Diese auf «kreative Weise» (Knaus 2018) zu adaptieren, wird dabei nicht als mechanischer Vorgang verstanden, weil mit einer kreativen Adaptation vorausgesetzt wird, dass etwas da ist, an das adaptiert werden kann. Insofern scheint für den Prozess der medienpädagogischen Interdisziplinarität eher die Metapher der besitzergreifenden Aneignung als die Metapher der Auflösung geeignet zu sein. Das zeichnet die medienpädagogische Bezugnahme auf Architektur, Medienwissenschaft, Kommunikationswissenschaft, Medienphilosophie, Medienpsychologie, Mediensoziologie und weitere Disziplinen aus. 
In der Medienpädagogik ist neben der Handlungsorientierung die Notwendigkeit einer Medienkritik kaum bestritten worden: Medienkritik wird als ein Kernproblem verstanden. Dass dabei verschiedene Kritikbegriffe argumentiert werden, ist Ausdruck des Umstandes, dass es einen Konsens über das zu diskutierende Problem gibt, von dem aus zumindest im deutschsprachigen Diskurs schon die Idee, Medienpädagogik auf bewahrpädagogische oder affirmative Positionen zu verpflichten, zurückgewiesen wird. Wie auch immer die Absicht der Medienkritik verstanden wird, ist klar, dass jede Medienkritik eine Distanzierung von Medien erfordert.

Eine auf die Bildung des Menschen bezogene Methode der Distanzierung wird als Übersetzungsproblem verstanden. Das Übersetzungsproblem wird derzeit in der Regel als Transformationsproblem bezeichnet. Dieser Sprachgebrauch wird hier übernommen.

Genau wie das Problem der Sprache verschiedene Sprachen impliziert, impliziert das Problem des Mediums verschiedene Medien, zwischen denen nur Transformationen vorgenommen werden können. Da es sich dabei um Transformationen zwischen Sprachen oder Medien handelt - etwa um eine Transformation von der Unterrichtssprache in die Wissenschaftssprache (Meder 1977, 24) entlang der Korrelation von Praxis und Theorie (Swertz und Mildner (Sontag) 2015, 8), um die Transformation von primären Erfahrungen in das didaktische Spiel der Simulationen (Meder 1995, 63), um die Transformation der Theatersprache in die Filmsprache (Meder 2008, 223) oder um die Transformation als Transmaterialisierung der Verhältnisse des Einzelnen zu Sachverhalten, anderen und sich selbst (Meder und Iske 2014, 80) - ist klar, dass diese Handlungen nicht als Transformationen in der Sprache verstanden werden können.

Ebenso können Transformationen zwischen Medien nicht als Transformationen in dem Medium verstanden werden, sondern nur als Transformationen zwischen Medien, denn: «Sprechen ist ein unvermitteltes Operieren mit Bedeutungen, d.h. in seiner Naturgebundenheit sinnbestimmt, in seiner Sinnbestimmtheit naturgebunden. [...] ich gehe in dieses Naturereignis ein» (Hönigswald 1937, 25f.). Genau und nur dieses Operieren produziert Transformationen, und es sind solche Operationen, in denen Akteurinnen und Akteure ihre Entscheidungsimpulse setzen und zeigen. 
Meder (2015) hat in einer Analyse der Technologie gezeigt, dass digitale Medien einen bildenden Gehalt haben. In diesem Sinne hat Damberger Augmented Reality als Anlass für Bildungserfahrungen- und -prozesse diskutiert und argumentiert, dass die Nutzung von Augmented Reality es ermöglicht, «einen Zugang zu den Repräsentationen der Spiegelungen der Welt in den Köpfen anderer Menschen zu erlangen» und das mit der Bildungsaufgabe verbunden, «sich selbst durch diese erweiterte Realität in Erfahrung zu bringen» (Damberger 2016, 23). Damberger zeigt damit, dass Augmented Reality als vorhandenes Medium in der Kommunikation von Akteurinnen und Akteuren mit sich selbst und mit anderen einen bildenden Gehalt aufweist.

Das kann daher für Künstliche Neuronale Netzwerke (KNN) auch vermutet werden. Daher wird hier mit dem bisher entwickelten Verständnis von Medienbildungsprozessen als Rezeptionsprozessen (gebildet werden) und als Produktionsprozessen (sich bilden) (Swertz 2017a; 2017b) im Rahmen der relationalen Medienpädagogik die Frage aufgeworfen, ob neben der Augmented Reality auch die datenbasierte Programmierung mit KNN als Thema in den Kanon der Fachdidaktik Medien, die mit dem Begriff der Medienkompetenz konzentriert wird, aufgenommen werden sollte. Dazu muss zunächst der Gegenstand veranschaulicht werden.

\section{Datenbasierte Programmierung}

Grundlage von Künstlichen Neuronalen Netzwerken (KNN) sind Perzeptrone (Abb. 1). Als Perzeptrone werden Simulationen von Nervenzellen bezeichnet. Simuliert werden die Axone, also die zu einer Nervenzelle führenden «Leitungen» als «Eingänge» einer Zelle, die Verarbeitung der «eingegebenen Daten» in der Zelle und die «Ausgabe». Mit Perceptronen werden dabei in der Regel viele untereinander «verschaltete» Nervenzellen simuliert. Das wird als künstliches neuronales Netzwerk bezeichnet. Mit solchen Modellen soll die Struktur des Gehirns abgebildet werden. 


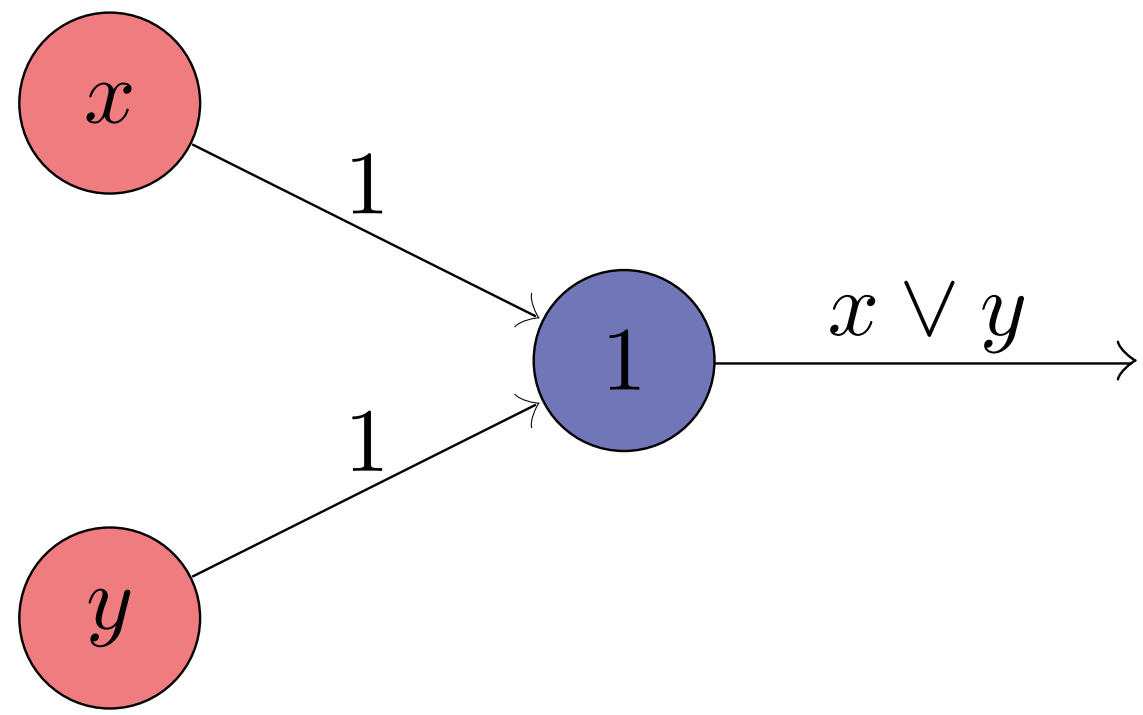

Abb. 1.: Perzeptron. Quelle: MartinThoma - Eigenes Werk, CC BY 3.0, https://commons.wikimedia.org/w/index.php?curid=28211506.

Perzeptrone wurden von McCulloch (dem Tagungsleiter der die Kybernetik begründenden Macy-Konferenzen) und Pitts als logische Schwellenwertelemente eingeführt, die Eingabevektoren in Ausgabevektoren umwandeln (McCulloch und Pitts 1943). Die prinzipielle Funktionsweise ist simpel: Wenn die Summe der Eingänge einen Schwellenwert überschreitet (also etwa grösser oder gleich 1 ist), wird auch der Ausgang aktiviert (also etwa auf 1 geschaltet). Wenn die Summe der Eingänge den Schwellenwert unterschreitet (also etwa kleiner als 1 ist), wird der Ausgang deaktiviert (also etwa auf o geschaltet). Perceptrone können auch aufwändiger modelliert werden, was an der Struktur aber nichts ändert.

Durch die Kombination solcher Perzeptrone können und-, oder- und nicht-Verknüpfungen realisiert werden. Rosenblatt (1958) hat gezeigt, dass mit einem mehrlagigen Perzeptron auch XOR - Verknüpfungen realisiert werden können. Die einen kontradiktorischen Gegensatz ausdrückende XOR-Verknüpfung (die dem Sheffersche Strich und dem Einheitsoperator nach Wittgenstein entspricht) ist dabei entscheidend, weil alle weiteren logischen Operationen aus dieser Operation abgeleitet werden können. Weil mit dieser Verknüpfung alle logischen Operationen realisiert werden können, ist klar, dass die Konstruktion einer Maschine, die diese Operation 
ausführt, es möglich macht, alle berechenbaren logischen Operationen maschinell auszuführen. Das wird auch als turingmächtige Maschine bezeichnet. Es gibt - kurz gesagt - keinen prinzipiellen Unterschied zwischen Computern und KNN.

Die Pointe bei KNN, die aus Perzeptronen bestehen ist, dass die Schaltung nicht fest verdrahtet wird, sondern die Gewichte an den Kanten zunächst zufällig initialisiert werden. Ausgangspunkt ist also nicht 1 oder O, sondern Rauschen. Nach der Initialisierung wird ein Eingabewert angelegt und die Berechnung der Ausgaben (Forward Propagation) ausgeführt. Die erste Ausgabe ist also beliebig. Anschliessend wird der erwartete Ausgabewert mit dem aus den Eingaben berechneten tatsächlichen Ausgabewert verglichen. Wenn die tatsächliche Ausgabe nicht der erwarteten Ausgabe entspricht, werden die Gewichte an den Kanten anhand der Differenz zwischen tatsächlicher und erwarteter Ausgabe in kleinen Schritten durch alle Ebenen von hinten nach vorn korrigiert. Dazu wird ein automatisches Verfahren verwendet. Diese automatische Korrekturverfahren wird als Backpropagation bezeichnet.

Backpropagation ist das, was in der Informatik als Lernen (machine learning) bezeichnet wird. Diese Wortwahl ist, wie Pias in seiner Interpretation der Arbeit von McCulloch schreibt, der kybernetischen Identifikation von «Synapsen oder von Röhren, [...] von Schaltern oder von Tintenstrichen» (Pias 2002, 55) geschuldet. Für McCulloch sind es nach Pias «Begriffe, die arbeiten und funktionieren, die zugleich theoretische wie praktische Entitäten begründen, die neuronale Strukturen modellieren und zugleich Artefakte konstruieren» (ebd.). Damit werden Begriffe als denkende Lebewesen betrachtet. Genau so argumentieren Jörissen und Verständig, wenn Sie schreiben:

«Ubiquitär vernetzte rechenbasierte Systeme nehmen nicht nur die Rolle von Entscheidungsträgern ein, sie werden im Sinne der Komplexitätsreduktion und verstärkten Einbettung von automatisierten Lösungen zugleich zu epistemischen Akteuren, die als lernende Netzwerke, ähnlich neurobiologischen Lernprozessen, hyperkomplexe Informationsverarbeitung betreiben und somit zum Akteur im gesellschaftlichen Wandel werden» (Jörissen und Verständig 2017, 40). 
Insofern «Werden» aus der hier vertretenen Perspektive nur dem Menschen zukommt, ist diese Sichtweise überraschend. Denn es ist offensichtlich, dass Sprache nicht spricht, sondern gesprochen wird. Begriffe formulieren sich auch nicht selbst und leben auch nicht, schon weil sie sich nicht vermehren können. Ebenso offensichtlich ist, dass Tote nicht lernen. Daher fällt das Backpropagationverfahren nicht unter den Begriff des Lernens, sondern unter den Begriff des Programmierens, der daher im Folgenden verwendet wird.

In der Anwendung von Backpropagationverfahren (für die es eine Vielzahl verschiedener Algorithmen gibt (Alpaydin 2020)), wird zwischen überwachter Programmierung, nicht überwachter Programmierung und verstärkender Programmierung unterschieden. Überwachte Programmierung bezeichnet den eben dargestellten Vergleich von berechneten mit erwarteten Ausgabedaten. Bei nicht überwachtem Programmieren wird der Vergleich nicht mit erwarteten Ausgabedaten, sondern mit bereits verarbeiteten Eingabedaten vorgenommen. Dadurch werden die Eingabedaten heuristisch klassifiziert. In der verstärkenden Programmierung werden Belohnungen und Bestrafungen nach Aktionen als Daten verwendet. In jedem Fall werden Eingaben und Ausgaben nicht durch logische Schlüsse (Algorithmen), sondern durch Wahrscheinlichkeiten verbunden, die im Zuge der Analyse der Daten erst berechnet werden. Es handelt sich also um eine datenbasierte Programmierung.

Eine Eigenschaft der datenbasierten Programmierung ist es, dass die Anpassungsschritte im Programmierprozess klein sind. Daher sind für die Programmierung viele Daten erforderlich. Dieser Datenbedarf wird derzeit als Big Data diskutiert. Big Data können nicht zuletzt wegen des effektiv skalierenden Backpropagationverfahrens überhaupt verarbeitet werden und sind zugleich für die datenbasierte Programmierung erforderlich. Die Diskussion über Big Data ist daher eine Nebenwirkung der effektiven Implementierung von Verfahren der datenbasierten Programmierung, die im Übrigen auch auf der Idee basiert, die Algorithmen in Grafikchips auszuführen, was interessante Bezüge zur Computerspieleforschung nahelegt.

Eine weitere Eigenschaft der datenbasierten Programmierung ist es, dass im Falle nicht linear separierbarer und nicht typisierter Daten nur eine Annäherung an das erwartete Ergebnis möglich ist. Auf eine 
bestimmte Eingabe erfolgt nicht immer die gleiche, logisch richtig geschlossene Ausgabe, sondern eine wahrscheinliche Ausgabe. Die Forwardpropagation liefert keine exakten Ergebnisse, sondern Wahrscheinlichkeiten, oder, im Sinne der Shannonschen Informationsentropie (Shannon und Weaver 1964), reduzierte Unsicherheit, aber keine Sicherheit. Ebenfalls relevant ist, dass eine explizite Modellierung eines spezifischen Problems für die datenbasierte Programmierung nicht erforderlich ist, weil mit dem Verfahren eine implizite Modellierung vorgenommen wird, die durch die Auswahl und die Gestaltung der Daten gesteuert wird.

\section{Medienpädagogische Analysen}

Nach dieser knappen Skizze geht es nun um die Frage, ob Backpropagation in der Fachdidaktik der Medien berücksichtigt werden sollte, ob der Gegenstand also dafür geeignet ist, Medienkompetenz zu vermitteln. Dass das vorher skizzierte Verfahren als Inhalt im Bereich der Medienkunde vermittelt werden kann, ist klar. Noch nicht klar ist, ob der Gegenstand auch für Medienkritik (Reflexion) und Mediengestaltung relevant ist. Dazu werden im Folgenden mögliche Inhalte skizziert.

\subsection{Reflexionen}

\subsubsection{Genres}

Eine Möglichkeit, Medien zum Zwecke der Reflexion zu distanzieren, sind Genrebegriffe. Eine Genretheorie für Räume, die mit turingmächtigen Maschinen konstruiert wurden, gibt es bisher allenfalls in Ansätzen. Eine im Sinne eines Thesaurus (also eines Verfahrens der Ordnung von Wissen durch Schlagworte, bei denen die zur Indexierung verwendeten Vokabeln aus vorhandenen Daten gewonnen und dann relationiert werden) entwickelte Ordnung, mit denen die datenbasierte Programmierung erfasst werden kann, kann an Wissenrepräsentationsmodellen oder an Programmiermodellen bzw. Programmierparadigmen orientiert werden. 
Als Wissensrepräsentationsmodelle werden derzeit logische Modelle (Programmlogik), Prozedurensysteme, Prädikatorenlogik (Deduktive Datenbanken), semantische Netze und neuronale Netze unterschieden. Als Programiermodelle werden prozedurale, funktionale, objektorientierte und stochastische Modelle (datenbasierte Programmierung) unterschieden. In beiden Fällen können neuronale Netze nicht einem anderen Genre zugeordnet zu werden, sondern stellen insbesondere wegen der auf dem Begriff der Informationsentropie basierenden stochastischen Basis ein eigenes Genre dar. Insofern die Kenntnis von Genretheorien ein relevantes Teilgebiet der Medienkunde ist und die datenbasierte Programmierung als eigenes Genre zu verstehen ist, ist das Thema KNN für die Vermittlung von Medienkompetenz relevant. Zudem erfordert die Produktion von Inhalten, die diesem Genre entsprechen, d.h. die praktische Verwendung von KNN zur Lösung von Problemen, wegen der Eigenständigkeit des Genres eine Transformation von Problemen in dieses Genre, so dass Übersetzungsprobleme entstehen.

\subsubsection{Mediengeschichte}

Neben Genretheorien ist zu bedenken, dass eine Grundlage jeder wissenschaftlichen Distanzierung der Akt der räumlichen Stillstellung der zeitlichen Wahrnehmung in einem Medium ist. Traditionell wird dazu der gedruckte Text verwendet, der als dauerhaft fixierte Lebensäusserung in der Hermeneutik paradigmatisch geworden ist. Mit der Entscheidung für gedruckte Texte wird eine spezifische Wahrnehmung von Geschichte verbunden. So können mit auf Papier gedruckten Texten zukünftige Menschen adressiert werden. Das haben McCulloch, Pitts und Rosenblatt getan. Ihre Arbeiten in den Blick zu nehmen, impliziert daher eine medienhistorische Perspektive, die einen relevanten Raum für Interpretationen öffnet. Dass es sich dabei um relevante Kontexte handelt, wurde bereits gezeigt.

Weil historische Interpretationen semiotische Prozesse erfordern, sind dabei immer verschiedene Lesarten möglich. Ein Beispiel für verschiedene Lesarten liefert die von Rosa (2005) aufgestellte Beschleunigungsthese. In seiner Argumentation führt Rosa Inhalte von Medien als Belege an, übersieht aber die Mediengeschichte der Technologie. Mit den 
mediengeschichtlichen Daten kann argumentiert werden, dass seit 1936 an Computern (Turing 1936) und seit 1943 an KNN im Prinzip nichts geändert wurde. Und weil Turings Vermutung, dass alles, was man berechnen kann, mit Computern auch berechnet werden kann, bewährt wurde, kann daran auch nichts geändert werden. Weil daher mit der ubiquitären Verbreitung von digitaler Technik Veränderungen erschwert werden, müssen Digitalisierungsprozesse als Entschleunigungsprozesse verstanden werden.

Für die Vermittlung von Medienkompetenz, in der Mediengeschichte als ein Aspekt der Medienkunde berücksichtigt wird, ist es relevant, solche unterschiedlichen Lesarten von Wahrnehmungen zu präsentieren, weil die Irritation Widerstand hervorrufen, Transformationen anregen und so zur Entwicklung eines eigenen Standpunkts motivieren kann. Dafür sind KNN, wie das Beispiel der Be- und Entschleunigung zeigt, geeignet.

\subsubsection{Hypes}

Neben der Entschleunigung kann auch der Begriff des Hypes zur Distanzierung von KNN verwendet werden. Hypes können als überhöhende Behauptungen definiert werden, die Aufmerksamkeit erzeugen. Einen Hype zu produzieren ist etwa Newell, Shaw und Simon (1959) mit dem General Problem Solver (GPS) gelungen. Das Genre der Prozedurensysteme ist zwar in der Praxis gescheitert, etwa in den pädagogisch relevanten Intelligenten Tutoriellen Systemen, die diskret entsorgt worden sind; die Story, die Newell, Shaw und Simon erzählt haben, hat aber so viel Aufmerksamkeit erzeugt, dass sich die Idee von Prozedurensystemen heute noch als prozedurales und problemlösendes Denken im Konzept der Digitalen Kompetenzen der Europäischen Kommission (Ferrari, Punie, und Brečko 2013), mit dem Menschen also als kybernetische Maschinen verstanden werden, oder in der Unterrichtspraxis in Form des Problem Based Learning (Shoemaker 1960) findet. Und auch die kognitive Psychologie ist eine Nebenwirkung dieses Hypes.

KNN werden nun wie der GPS verwendet, um einen Hype zu erzeugen. Damit wird deutlich, dass KNN wie einige andere informatische Konzepte gute Beispiele zur Illustration der Agenda-Setting-Theorie (Cohen 1963) liefern, nach der mit Medien bestimmt wird, über welche Themen Menschen 
sich Gedanken machen. Insofern sind KNN für die Medienkompetenzvermittlung exemplarisch relevant. Auf ähnlichem Weg findet sich das kybernetische Konzept des Feedback - und Backpropagationverfahren sind Feedbackverfahren - zum Beispiel in Hatties Studie zu Visible Learning (Hattie 2018) als zentraler Begriff für die Beschreibung erfolgreichen Lehrpersonenhandelns wieder. Damit wird Lehrpersonenhandeln von Hattie als kybernetischer Prozess begriffen, und nicht als Beziehung, Verständigung oder kommunikatives Handeln. Die ideologischen Implikationen sind offensichtlich. Mit Feedback ist die optimale Regelung Lernender und nicht der dornichte Pfad der Kritik gemeint. KNN liefern damit einen Anlass, Medienkompetenz im Sinne von Baacke selbstreflexiv, d.h. im Wege der Reflexion des Unterrichtshandelns im Unterricht zu vermitteln.

\subsubsection{Mediensozialisation}

Die mit dem Feedback wohl unbedacht vorgenommene Transformation von Technologie in Erziehungsziele, didaktische Prinzipien und Bildungsideale lassen eine Analyse der Erziehungswissenschaft aus Sicht von Mediensozialisationstheorien sinnvoll erscheinen. Dafür muss hier ein Beispiel genügen: In der Erziehungswissenschaft haben Wimmer und Schäfer das Infragestellen jeglicher Gewissheit zum Ausgangspunkt ihrer Untersuchungen zur Selbstauslegung im Anderen gemacht (Wimmer und Schäfer 2006) und damit unbedacht den Ansatz, den Shannon und Weaver (1964) mit dem Begriff der Informationsentropie gemacht haben, übernommen. Das ist daran zu erkennen, dass Wimmer und Schäfer das Infragestellen erodierter Zugehörigkeiten, stabiler Identitäten und aller Grundkoordinaten kultureller Selbstverortung diagnostizieren; sie interpretieren diese Prozesse anschliessend mit der Fremderfahrungstheorie.

Aus Sicht einer Mediensozialisationstheorie kann die Diagnose des Endes jeder Gewissheit auch als Geschmack, als Ausdruck eines medialen Habitus (Kommer 2010) interpretiert werden. Es handelt sich um einen Fall, in dem Menschen, die in ihrer Mediensozialisation den für eine Buchdruckkultur typischen medialen Habitus erworben haben, mit der Wahrnehmung simulierter Welten konfrontiert werden, was nicht ihrem Geschmack entspricht. 
Diese Welten sind simuliert, weil sie mit turingmächtigen Maschinen (und nicht mit Buchdruckpressen) produziert werden. Weil aber alles, was berechnet werden kann, simuliert werden kann, und dabei die Simulation turingmächtiger Maschinen eingeschlossen ist, wird mit den Simulationen bei Menschen, die einen für die Buchdruckkultur typischen medialen Habitus aufweisen, der Eindruck von Pluralität erzeugt, vom Ende jeder Gewissheit.

Turingmächtige Maschinen bedeuten in der Tat das Ende des von Comenius angesichts des Buchdrucks formulierten pansophischen Gedankens - wenn nur die Oberfläche betrachtet wird. Simuliert werden alle diese Welten aber von der immer gleichen Maschine, die oberflächlich plural ist, in der Tiefe aber auf nur einer einzigen logischen Operation basiert. Aus dieser Sicht können wir derzeit häufig die Produktion eines medialen Habitus beobachten, den Menschen erwerben, die an der weltweit technisch vereinheitlichen digitalen Kultur teilnehmen. Und das ist, weil die Möglichkeiten von Computern sich weder ändern können noch geändert werden können, nicht plural, sondern singulär, stabil und heteronom.

\subsubsection{Räume}

Auf solche Tendenzen der Kommunikation hat bereits Innis (1951) aufmerksam gemacht und argumentiert, dass Medien Wissen entweder gut im Raum und schlecht in der Zeit oder schlecht im Raum und gut in der Zeit transportieren: Die Übergabe der 10 Gebote auf einem USB-Stick bedeutet keine ewige Wahrheit - dafür braucht es in Stein gemeisselten Text. Die mediale Temporalität legt nach Innis Verschiebungen gesellschaftlicher Machtverhältnisse nahe, und zwar insbesondere zwischen weltlichen und religiösen Mächten.

An Backpropagationsverfahren kann nun die räumliche Tendenz der Computertechnologie gezeigt werden, denn diese Verfahren haben kein Gedächtnis, sondern einen Speicher. Daher können mit KNN nur Ausgaben auf Grundlage von in der Gegenwart anliegenden Eingaben berechnet werden. Berechnet werden kann dabei in der als Vorhersage deklarierten Ausgabe nur, was in den bei der Programmierung angelegten Daten bereits geschehen ist. Zukunft und Vergangenheit werden so ausgeblendet. 
Genau solche mit dem Medium Computer einhergehenden Tendenzen zur Betonung der Kommunikation im Raum sind es, die für Menschen, für die der Buchdruck die dominante Sozialisationsinstanz bei der Entwicklung ihres medialen Habitus war, irritierend sind, was dann zur These des Infragestellens aller Grundkoordinaten kultureller Selbstverortung verleitet. Die gleichzeitig stattfindende weltweite Normierung dieser Grundkoordinaten mit digitalen Technologien wird übersehen und so in paranoischer Manier (Barberi 2018, 84) das eigene Panopticon (Foucault 1993) gebaut.

Diese Reflexion zeigt, dass mit Backpropagationverfahren die reflexive Anwendung analytischen Wissens auf sich selbst (Baacke 1997) motiviert werden kann. Daher kann reflexiv distanzierende Medienkritik als eine Dimension der Medienkompetenz an diesem Gegenstand vermittelt werden.

\subsubsection{Identität}

In einer dritten Distanzierung ist es interessant, Neuronale Netzwerke als kybernetische Maschinen zu betrachten, in denen die XOR-Verknüpfung der Steuermann ist. Für die Erziehungswissenschaft ist dazu an die Auseinandersetzung um die kybernetische Didaktik zu erinnern, die von Cube (1965) vorgelegt hat. Pongratz fasst 1978 seine Kritik an der Kybernetik und den kybernetischen Systemtheorien so zusammen:

«Gefordert ist die reduktive Elimination des Subjekts, sein Ersatz durch die technische Objektivation. Dieser Reduktionsprozess aber kann die dem Subjekt zugehörige Dimensionen der Geschichte, der Freiheit und Verantwortlichkeit sich nicht unbeschadet anverwandeln» (Pongratz 1978, 255).

Diesen Tendenzen stellt Pongratz in einer negativen Anthropologie die Freiheit des Subjekts entgegen, sich zu sich selbst und zur Welt selbst in ein Verhältnis zu setzen (ebd., 29).

Die Möglichkeit, Freiheit bei all dem Zwange zu entwickeln, ist seitdem in Abrede gestellt worden. So halten Wimmer und Schäfer im Anschluss an Derrida fest: «Was diese erste und eigene Sprache [gemeint ist die Muttersprache] zu sagen untersagt, ist die Einzigartigkeit des Eigenen, des 
Selbst» (Wimmer und Schäfer 2006, 16). Daher wird die Möglichkeit der symmetrischen Anerkennung autonomer Subjekte als immer schon herrschaftsförmig und gewalttätig ausgewiesen.

Dieses Problem betrifft kybernetische Maschinen nicht. Auch wenn, wie Meyer-Drawe bemerkt hat, von Menschen produzierte Maschinen menschliche Selbstbeschreibungen begründen (Meyer-Drawe 1996, 28), ist klar, dass Maschinen niemals den Status des von Wimmer und Schäfer beschriebenen Fremden erreichen können, weil sie nur aus Kalkülzeichen bestehen und es daher - auch im Sinne hermeneutischer Sinnprozesse keine Semiose geben kann (Swertz 2000, 197f.).

Zwar könnte man meinen, dass neuronale Netzwerke eine Fremdauslegung erzeugen. Dass ist aber allenfalls die Simulation einer Fremdauslegung und insofern eine Illusion (Sesink 2004). Maschinen können gegenüber Menschen die Illusion von Autonomie erzeugen, was auch in der Rede von autonomen Systemen zum Ausdruck kommt. Demgegenüber konnten Menschen autonom sein - zumindest bis Autonomie als eine Funktion der als Lebewesen halluzinierten Sprache verstanden wurde, so dass Autonomie nichts anderes mehr sein konnte als eine Reproduktion von in der Sprache vorhandenen Strukturen.

Eine solche Reproduktion ist mit Neuronalen Netzwerken möglich und wird z.B. im transhumanistischen Diskurs stark gemacht, was die Frage aufwirft, wie Menschen dazu angeregt werden können, sich aus diesen Technologien der Macht herauszudrehen. Schäfer (2004) bedenkt in diesem Zusammenhang zwar nicht explizit, aber implizit das Problem, dass eine solche Machtkritik wegen des Unentrinnbarkeitsarguments einen universalisierenden Anspruch erheben muss, damit selbst in den kritisierten Bereich fällt und sich so selbst widerspricht. Schäfer schlägt daher vor, das Verhältnis zwischen Singularität und symbolisch angebbarem Selbst als das zu verstehen, innerhalb dessen man noch gar kein Selbst hat. Dieses Verhältnis ist dann eine vor allen empirischen Erfahrungen liegende Bedingung der Möglichkeit der Produktion des Selbst - das abstrakte «Ich denke» und das empirische "Ich bin» bleiben unterschieden; die Vorstellung der Identität des Subjekts bleibt empirisch und führt nicht zur Auflösung der Dialektik. Kurz: Eine souveräne Reflexion von Technologie und eine souveräne Gestaltung von Technik bleiben möglich. 
Weil Maschinen im Unterschied zu Menschen immer vollständig mit sich selbst identisch sind, ist es so absurd zu sagen, dass Maschinen denken oder lernen, dass zu fragen ist, wie Menschen dazu gebracht werden können zu sagen, dass Maschinen denken oder lernen. Das drängt eine ideologiekritische Analyse auf.

\subsubsection{Ideologiekritik}

Den marktradikalen Kapitalismus mit dem amtierenden Papst als lebendigen Teufel anzuprangern, ist zwar ebenso in Mode wie Pisa-Bashing. Daran zu erinnern, dass mit Systemen, mit denen Durchschnittwerte durchgesetzt werden, Menschen, die nicht durchschnittlich sein möchten, ausgegrenzt werden und Menschen, die nicht durchschnittlich sein können, marginalisiert werden, ist dennoch erforderlich. Die Durchschnittsorientierung - und Backpropagationverfahren berechnen nichts anderes als Durchschnitte - nötigt Menschen zu einer Anpassung an die Maschine und dient so zur Durchsetzung von Interessen.

Das perpetuiert durchaus Vertrautes: Manipulation, Ausbeutung, Entfremdung und Unterdrückung. Und wie das funktioniert, kann anhand von Anwendungen des Backpropagationverfahrens bei kapitalistischen und politischen Institutionen wie Amazon oder der NSA gut gezeigt werden. Das macht nochmals deutlich, dass Backpropagationverfahren geeignete Exempel sind, um analytisch problematische gesellschaftliche Prozesse zu erfassen und so Medienkritik in einem exemplarischen Unterricht zu vermitteln, ohne - und darum geht es hier - auf die souveräne Gestaltung zu verzichten.

Das Backpropagationverfahren bietet damit relevante Anlässe für eine reflektierende Distanzierung. Relevant sind diese Anlässe, weil die Technologie von Menschen dazu verwendet wird, ihre Verhältnisse zu anderen, zur Welt und zu sich selbst zu gestalten. Die Reflexion von Verfahren anzuregen, mit denen Menschen ihre Identität gestalten, sich also die selbst erzeugten Subjektivierungsbedinungen klar zu machen und umzugestalten, hat einen Wert für die Bildung des Menschen. 


\subsection{Produktionen}

Nun stellt sich das Problem, dass Medienkritik und Medienkunde noch keine Handlungsoptionen zeigen können. Allerdings sind Akte der Selbstermächtigung im Sinne einer «Diskursproduktion als Zeichenproduktion» (Barberi 2018, 146) durchaus möglich, wenn die vorherige Argumentation als ein Akt der Distanzierung akzeptiert werden kann.

Damit rückt die Entwicklung von Designs mit Backpropagationverfahren in den Blick. Hier ist zunächst der Orientierungsbegriff relevant. Orientierungswissen ist auch in der Medienpädagogik als Zielbegriff medienpädagogischen Handelns verwendet worden (Marotzki 1990). Nun hat Mittelstrass, auf den dabei gelegentlich verwiesen wird, in seinem Aufsatz zu Glanz und Elend der Geisteswissenschaften argumentiert, dass Orientierungswissen nichts anderes liefert als Kompensationswissen: «Die Geisteswissenschaften kompensieren Modernisierungsschäden, indem sie erzählen» (Mittelstraß 1989, 12). Das wirft z.B. die Frage auf, ob nicht narratologische Analysen von Biografien vor allem dazu beitragen, Modernisierungsschäden zu kompensieren - etwa auf dem Wege der Biografiearbeit.

Um nun eine blosse Kompensationsfunktion zu vermeiden, ist es nach Mittelstraß nötig, die Zukunft zu gestalten. Das erinnert an Litts Auseinandersetzung mit dem Problem von Führen und Wachsenlassen (Litt 1927) oder an Hönigswald Begreifen der «Zukunft als pädagogischer Idee» (Hönigswald 1927, 108). Interessanterweise wird dieses Problem in der Medienpädagogik aber kaum als Zukunftsproblem diskutiert, sondern als Unvollständigkeitsproblem: Baacke hat seine erkenntnistheoretische Wendung des Metakommunikationsbegriffs, mit der er die metakommunikative Medienpädagogik fundiert hat, mit der Russelschen Antinomie begründet (Baacke 1973). Ganz ähnlich hat Meder die relationale Medienpädagogik im Rückgriff auf den Gödelschen Unvollständigkeitssatz begründet (Meder 2004). Gödel war ebenso wie Turing und Wittgenstein ein Schüler Russels (vgl. zur Pädagogik Russels (Swertz 2020)) und hat zeitgleich mit Shannon in Princeton gearbeitet. In beiden Ansätzen wird damit die Begrenztheit der Vernunft zum Augangspunkt gemacht. Eine Antwort darauf, wie diese Grenze im Blick auf die Freiheit bei all dem Zwange thematisiert werden kann, ist das Hervortreten des Spieltriebs aus Stoff- und Formtrieb. 
Dieser Gedanke Schillers wird in der relationalen Medienpädagogik mit einem reformulierten Ästhetikbegriff (Meder 1997) und in der metakommunikativen Medienpädagogik mit dem Kreativitätsbegriff als vierter Dimension des Medienkompetenzbegriffs (Baacke 1996) aufgegriffen. Mit ästhetisch motivierter Kreativität geht es nicht nur darum, die Welt zu verstehen oder in subversiven Akten Widerstand zu leisten, sondern auch darum, die Welt zu gestalten. Das wird in der relationalen Medienpädagogik als undogmatisches Dogma markiert:

«Die ästhetische Darstellung [...] ist Produktion einer präsenziellen Gestalt und grenzt sich darin vom wissenschaftlichen Erkennen ab» (Meder 1997, 19).

Das ist nicht logisch, aber schön - und zeigt die Möglichkeit der Orientierung pädagogischen Handelns an der Zukunftsoffenheit in der Gegenwart, die mit der Unvollständigkeit von Wissen begründet wird.

Wenn allerdings das «Innere» der Sprache - also Grammatik und Syntax - nur als stummes oder gänzlich unbewusstes Fungieren gedacht wird und insofern überhaupt nicht aussprechbar ist, drängt sich die Frage auf, ob das Innere dann in den Gegenstandsbereich des Begriffs der Sprache fallen kann. Wäre das nicht der Fall, würde Sprache aus sich selbst heraus sprechen und sich als Regel selbst setzen und regeln. Das ist aber, um das noch einmal zu betonen, nicht der Fall.

Daher ist es klarer, von Subjekten zu sprechen, die sich mit und in der Sprache ausdrücken können, auch wenn das nie vollständig möglich ist, weil das Subjekt nicht einfach nur Sprache ist. Ein Subjekt muss durchaus den Strukturen der Sprache entsprechen, produziert diese aber gleichzeitig. Dieser korrelative Bezug von Sprache und Subjekt kann nicht logisch, sondern nur ästhetisch in Ganzheit überführt werden (Meder 2014, 46). Genau das begründet eine handlungsorientierte Medienpädagogik. Die handlungsorientierte Medienpädagogik kann eine schöne medienpädagogische Gestaltung von Backpropagationverfahren orientieren. Die Entwicklung von Designs mit dem Backpropagationverfahren kann damit als eine Aufgabe für die Medienpädagogik verstanden werden. 
Dafür bietet sich der Selbstversuch an, indem etwa Backpropagationverfahren als Instrumente für die Analyse medienbiografischer Erzählungen verwendet werden. So die Produktion der Produktionsbedingungen erziehungswissenschaftlicher Erkenntnis in die Hand zu nehmen (Swertz 2017b), die Struktur von Backpropagationverfahren für die Analyse pädagogischen Verhaltens also in pädagogischer Absicht zu gestalten, erscheint als eine reizvolle Aufgabe - vor allem, weil mit Backpropagationverfahren Daten ohne eine vorhergehende Einschränkung durch die Formulierung von Variablen kategorisiert werden können. Das könnte gut zum Forschungsstil der Grounded Theory passen, denn die mit Zufallswerten initialisierten Netzwerke kommen ohne Vorerfahrungen aus und können qualitative Daten rein induktiv ordnen.

Eine zweite Möglichkeit ist es, die Kompetenz zur Verwendung von Backpropagationverfahren im Sinne der handlungsorientierten Medienpädagogik im Unterricht zu vermitteln. Wer sich nicht den von Facebook und Google trainierten Netzwerken überlassen möchte, kann selbst Backpropagationverfahren zur Filterung von Nachrichten gestalten und Neuronale Netzwerke trainieren. Die eigene Produktion einer Filterblase bedeutet aber ein ganz anderes Verhältnis zur Welt als die Nutzung manipulativ parametrisierter kommerzieller Filteralgorithmen. Mit eigenen Filtern können der eigene Geschmack und die eigene Vorstellung von Schönheit zum Ausdruck gebracht werden. Der Algorithmus wird zum Pinsel, mit dem Menschen sich selbst ausdrücken können. Mit solchen Praktiken wird das Eigentum an der Steuerung der eigenen Aufmerksamkeit und an den eigenen Handlungen, d.h. an den eigenen Daten, angeeignet.

Abschliessend kann kurz darauf verwiesen werden, dass gut handhabbare Beispiele und Entwicklungsumgebungen durchaus zur Verfügung stehen. Beispiele finden sich z.B. in der aktuellen Ausstellung im Ars Electronica Center in Linz. So zeigt etwa Abb. 2 die Betrachtung der Ausgabe eines neuronalen Netzwerks durch Besucherinnen und Besucher; der Tisch im Vordergrund dient der Eingabe von Mustern, die Bildschirme zeigen die Datenaufbereitungsschritte und rechts unten (erkennbar an den beiden weissen Punkten unter der grünen Überschrift) die Ausgabe des KNN. Eine Transformation von der Museums- in die Schulpädagogik steht zwar noch aus, erscheint aber nicht wirklich herausfordernd. Eine dafür 
geeignete Auswahl von Entwicklungsumgebungen findet sich etwa unter https://ai.google/education/. Dort werden auch Tutorials angeboten, auf die in einer medienpädagogisch fundierten Lernumgebung zurück gegriffen werden kann.

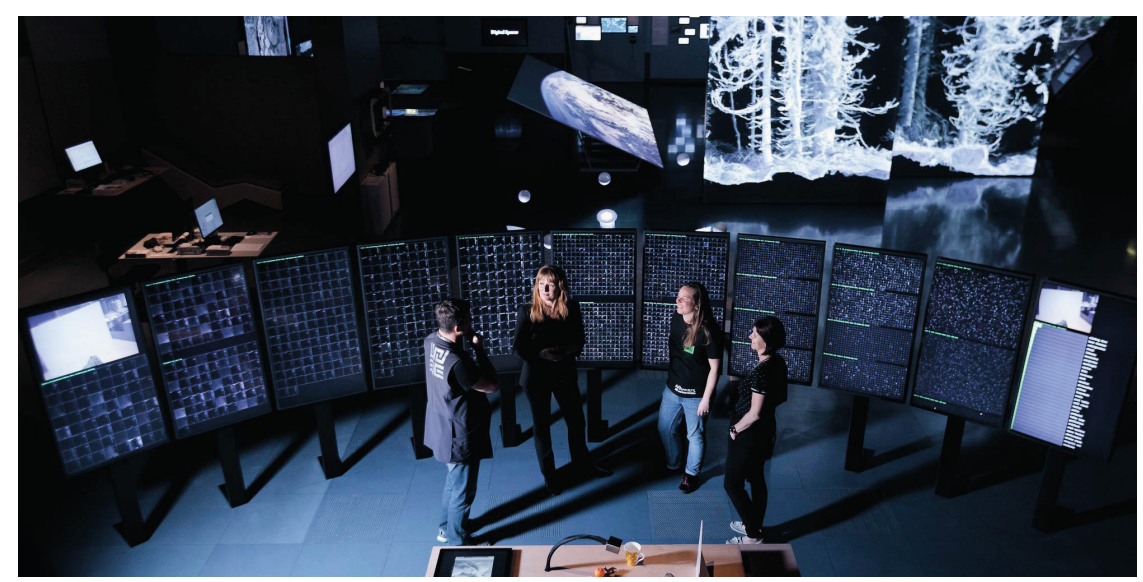

Abb. 2.: Inszenierung eines künstlichen neuronalen Netzwerkes im Ars Electronica Center in Linz. Quelle: Florian Voggeneder für Ars Electronica Center (https://ars.electronica.art/center/files/2019/05/exhibitions19.jpg ๑).

\section{Fazit}

Es wurde gezeigt, dass ein souveräner und reflektierter Umgang mit Medien am Beispiel von Backpropagationverfahren so vermittelt werden kann, dass Selbstreflexion angeregt und Selbstgestaltung motiviert wird. Dafür ist es erforderlich, die Zeichenstrukturen und die materialen Strukturen von künstlichen neuronalen Netzwerken als Medium kritisch zu reflektieren und kreativ zu gestalten.

Auf diesem Weg ist es möglich, von der Bedienung der Maschine dazu überzugehen, sich der Maschinen zu bedienen. Das steht im Kontext der Aneignung von Produktionsmitteln, der Selbstbestimmung, der Emanzipation und der Mündigkeit. Nicht diskutiert wurde die damit verbundene Notwendigkeit der Selbstbeherrschung, die den Begriff der Gemeinschaft, auf den der Begriff des Subjekts korrelativ bezogen ist, verweist. Die damit verbundenen Präferenzen für einen demokratischen Staat (Barberi und Swertz 2017) und eine solidarisch-liberale Ökonomie (Swertz 2012) 
wurden an anderer Stelle diskutiert. Werden diese Präferenzen akzeptiert, kann es nicht darum gehen, Menschen einen souveränen und reflektierten Umgang mit künstlichen neuronalen Netzwerken vorzuschreiben. Wer sich dafür entscheiden mag, als Maschinensklave zu leben, soll das tun. Unmoralisch (Baacke 1973, 305) ist es aber, die Möglichkeit eines freien Lebens nicht wenigstens zu zeigen. Die exemplarische Berücksichtigung von Backpropagationverfahren im Sinne einer handlungsorientierten Medienkompetenzvermittlung ist damit sinnvoll, und angesichts des aktuellen Hypes auch erforderlich.

\section{Literatur}

Alpaydin, Ethem. 2020. Introduction to Machine Learning. 4th edition. Cambridge, Massachusetts: MIT Press.

Baacke, Dieter. 1973. Kommunikation und Kompetenz: Grundlegung einer Didaktik der Kommunikation und ihrer Medien. München: Juventa-Verlag.

Baacke, Dieter. 1996. «Medienkompetenz als Netzwerk». medien praktisch 2: 4-10.

Baacke, Dieter. 1997. Medienpädagogik. Grundlagen der Medienkommunikation 1. Tübingen: Niemeyer.

Bachmair, Ben. 2005. «Qualität des Kinderfernsehens in einer von Alltagsästhetik bestimmten Kultur». Televizion 18 (2): 78.

Bachmair, Ben. 2017. «Mobilität - Innovationsanlässe für Pädagogik und Medienpädagogik in einer disparaten Kultur». Herausgegeben von Christian Swertz, Wolfgang B. Ruge, Alexander Schmölz, und Alessandro Barberi. MedienPädagogik: Zeitschrift für Theorie und Praxis der Medienbildung 29 (Konstitution der Medienpädagogik): 195-215. https://doi.org/10.21240/mpaed/29/2017.09.07.X.

Barberi, Alessandro. 2018. «Performanz und Medienkompetenz: Dieter Baackes Grundlegung der Medienpädagogik als Diskurspragmatik». Aachen: RWTH Aachen.

Barberi, Alessandro, und Christian Swertz. 2017. «Strukturwandel der Öffentlichkeit 3.0 mit allen Updates». In Der neue Strukturwandel der Öffentlichkeit, herausgegeben von Ulrich Binder und Jürgen Oelkers, 151-79. Weinheim: Beltz.

Baudry, Jean-Louis. 1994. «Das Dispositiv: Metapsychologische Betrachtungen des Realitätseindrucks». Übersetzt von Max Looser. Psyche 11 (48): 1047-74.

Cohen, Bernard Cecil. 1963. Press and Foreign Policy. Princeton University Press.

Cube, Felix von. 1965. KYpernetische Grundlagen des Lernens und Lehrens. Erziehungs-wissenschaftliche Bücherei. Stuttgart: Klett.

Damberger, Thomas. 2016. «Augmented Reality als Bildungsenhancement?» Medienimpulse 54 (1). https://journals.univie.ac.at/index.php/mp/article/view/ mi893. 
Damberger, Thomas, und Stefan Iske. 2017. «Quantified Self aus bildungstheoretischer Perspektive». In Das umkämpfte Netz, herausgegeben von Ralf Biermann und Dan Verständig, 17-36. Wiesbaden: Springer Fachmedien Wiesbaden. https://doi.org/10.1007/978-3-658-15011-2_2.

Ferrari, Anusca, Yves Punie, und Barbara N. Brečko. 2013. DIGCOMP: A Framework for Developing and Understanding Digital Competence in Europe. Luxembourg: Publications Office of the European Union. http://dx.publications.europa. eulo.2788/52966.

Foucault, Michel. 1993. Überwachen und Strafen: Die Geburt des Gefängnisses. Übersetzt von Walter Seittler. Frankfurt am Main: Suhrkamp.

Hattie, John. 2018. Visible Learning: Feedback. Boca Raton: Routledge. https:// www.taylorfrancis.com/books/9780429485480.

Hemmerich, Fabian. 2017. «Anmerkungen zu angstauslösenden Potenzialen fiktionaler Filme und Fernsehsendungen und pädagogischen Implikationen in der frühen Kindheit». Medienimpulse 55 (4): 1-50.

Hönigswald, Richard. 1927. Über die Grundlagen der Pädagogik. Ein Beitrag zur Frage des pädagogischen Universitäts-Unterrichts. 2. umgearbeitete Auflage. München: Ernst Reinhardt.

Hönigswald, Richard. 1937. Philosophie und Sprache. Basel: Haus zum Falken Verlag.

Hüther, Jürgen, und Bernd Podehl. 2017. «Geschichte der Medienpädagogik». In Grundbegriffe Medienpädagogik, herausgegeben von Bernd Schorb, Anja Hartung-Griemberg, und Christine Dallmann, 117-24. München: kopaed.

Innis, Harald Adams. 1951. The Bias of Communication. Toronto: University of Toronto Press.

Jörissen, Benjamin. 2016. «Zur bildungstheoretischen Relevanz netzwerktheoretischer Diskurse». In Von der Bildung zur Medienbildung, herausgegeben von Dan Verständig, Jens Holze, und Ralf Biermann, 231-55. Wiesbaden: Springer Fachmedien Wiesbaden. https://doi.org/10.1007/978-3-658-10007-0_12.

Jörissen, Benjamin, und Winfried Marotzki. 2009. Medienbildung - Eine Einführung: Theorie - Methoden - Analysen. 1. Aufl. Bad Heilbrunn: UTB GmbH.

Jörissen, Benjamin, und Dan Verständig. 2017. «Code, Software und Subjekt: Zur Relevanz der Critical Software Studies für ein nicht-reduktionistisches Verständnis "digitaler Bildung"». In Das umkämpfte Netz, herausgegeben von Ralf Biermann und Dan Verständig, 37-50. Wiesbaden: Springer Fachmedien Wiesbaden. https://doi.org/10.1007/978-3-658-15011-2_3.

Knaus, Thomas Hrsg. 2018. Forschungswerkstatt Medienpädagogik. https://doi. org/10.25526/fw-mp.28.

Kommer, Sven. 2010. Kompetenter Medienumgang? Eine qualitative Untersuchung zum medialen Habitus und zur Medienkompetenz von SchülerInnen und Lehramtsstudierenden. Budrich UniPress. https://doi.org/10.3224/94075539.

Litt, Theodor. 1927. Führen oder Wachsenlassen Eine Erörterung des Pädagogischen Grundproblems. Leipzig: B. G. Teubner. 
Marotzki, Winfried. 1990. Entwurf einer strukturalen Bildungstheorie. Biographietheoretische Auslegung von Bildungsprozessen in hochkomplexen Gesellschaften. Weinheim: Deutscher Studien Verlag.

Marx, Karl. 1962. Das Kapital. Band I - Kritik der politischen Ökonomie. Bd. 23. Karl Marx - Friedrich Engels - Werke. Berlin: Dietz Verlag. http://www.mlwerke.de/ $\mathrm{me} / \mathrm{me23} / \mathrm{me23}$ _00o.htm.

McCulloch, Warren S., und Walter Pitts. 1943. "A Logical Calculus of the Ideas Immanent in Nervous Activity». The Bulletin of Mathematical Biophysics 5 (4): 115-33.

Meder, Norbert. 1977. «Ansätze zur Prozeßanalyse des Unterrichts.»In, herausgegeben von Jürgen Grzesik, 21-75. Opladen: Leske und Budrich. https://doi. org/10.1007/978-3-322-88202-8.

Meder, Norbert. 1995. «Didaktische Überlegungen zu einem veränderten Unterricht durch den Einsatz neuer Technologien.» In Kommunikative Kompetenz in einer sich verändernden Medienwelt, herausgegeben von Jürgen Lauffer und Ingrid Volkmer, 48-63. Schriftenreihe der Gesellschaft für Medien und Kommunikationskultur in der Bundesrepublik e.V, Bd. 9. Opladen: Leske + Budrich.

Meder, Norbert. 1997. «(Ethik und Aesthetik sind Eins)». In Freizeit zwischen Ethik und Ästhetik, herausgegeben von Johannes Fromme und Renate Freericks, 15-35. Neuwied/Kriftel/Berlin: Luchterhand.

Meder, Norbert. 2004. Der Sprachspieler. Der postmoderne Mensch oder das Bildungsideal im Zeitalter der neuen Technologien. 2. wesentlich erweiterte Auflage. Würzburg: Königshausen und Neumann.

Meder, Norbert. 2008. «Walter Benjamin». In Handbuch Medienpädagogik, herausgegeben von Uwe Sander, Friederike von Gross, und Kai-Uwe Hugger, 1. Auflage, 217,223. Wiesbaden: VS, Verlag für Sozialwissenschaften.

Meder, Norbert. 2014. «Das Medium als Faktizität der Wechselwirkung von Ich und Welt (Humboldt)». In Perspektiven der Medienbildung, herausgegeben von Winfried Marotzki und Norbert Meder, 45-69. Wiesbaden: Springer Fachmedien Wiesbaden. https://doi.org/10.1007/978-3-658-03529-7_3.

Meder, Norbert. 2015. «Neue Technologien und Erziehung/Bildung». Medienimpulse 53 (1): 1-12. https://journals.univie.ac.at/index.php/mp/article/view/mi788.

Meder, Norbert. 2016. «Philosophische Grundlegung von Bildung als einem komplexen Relationengefüge». In Von der Bildung zur Medienbildung, herausgegeben von Dan Verständig, Jens Holze, und Ralf Biermann, 179-210. Wiesbaden: Springer Fachmedien Wiesbaden. https://doi.org/10.1007/978-3-658-10007O_10.

Meder, Norbert, und Stefan Iske. 2014. «Zur Empirie von Prozessen in der Bildungsforschung». In Arbeit am Begriff der Empirie, herausgegeben von Alfred Schäfer und Christiane Thompson, 79-99. Halle: Martin-Luther-Universität Halle-Wittenberg.

Meyer-Drawe, Käte. 1996. Menschen im Spiegel ihrer Maschinen. Paderborn: Wilhelm Fink Verlag. 
Mittelstraß, Jürgen. 1989. «Glanz und Elend der Geisteswissenschaften». Universität Oldenburg. http://oops.uni-oldenburg.de/1192/1/ur27.pdf.

Newell, Allen, J. C. Shaw, und Herbert A. Simon. 1959. «Report on a general problem-solving program.» P-1584. Santa Monica: Rand Coporation. http://bitsavers.informatik.uni-stuttgart.de/pdf/rand/ipl/P-1584_Report_On_A_General_Problem-Solving_Program_Feb59.pdf.

Pias, Klaus. 2002. «Die kybernetische Illusion». In Medien in Medien, herausgegeben von Claudia Liebrand und Irmela Schneider, 51-66. Köln: DuMont.

Pongratz, Ludwig A. 1978. Zur Kritik kybernetischer Methodologie in der Pädagogik: e. paradigmat. Kap. szientist. Verkürzung pädag.-anthropolog. Reflexion. Europäische Hochschulschriften: Reihe 11, Pädagogik, Bd. 58. Frankfurt am Main; Bern; Las Vegas: Lang.

Rosa, Hartmut. 2005. Beschleunigung. Die Veränderung der Zeitstrukturen in der Moderne. 11. Aufl. Frankfurt am Main: Suhrkamp Verlag.

Rosenblatt, F. 1958. «The Perceptron: A Probabilistic Model for Information Storage and Organization in the Brain.» Psychological Review 65 (6): 386-408. https://doi.org/10.1037/ho042519.

Schäfer, Alfred. 2004. «Alterität: Überlegungen zu Grenzen des pädagogischen Selbstverständnisses». Zeitschrift für Pädagogik 50 (5): 706-26.

Schiefner-Rohs, Mandy. 2012. Kritische Informations- und Medienkompetenz: theoretisch-konzeptionelle Herleitung und empirische Betrachtungen am Beispiel der Lehrerausbildung. Internationale Hochschulschriften 566. Münster: Waxmann.

Schorb, Bernd. 2009. «Gebildet und kompetent. Medienbildung statt Medienkompetenz?» Medien + Erziehung 53 (5): 50-56.

Sesink, Werner. 2004. In-formatio. Die Einbildung des Computers. Beiträge zur Theorie der Bildung in der Informationsgesellschaft. Bd. Band 3. Bildung und Technik. Münster: Lit Verlag.

Shannon, Claude, und Warren Weaver. 1964. The Mathematical Theory of Communication. Urbana: University of Illinois Press.

Shoemaker, Harry A. 1960. «The Functional Context Method of Instruction». IRE Transactions on Education 3 (2): 52-57. https://doi.org/10.1109/TE.1960.4322128.

Swertz, Christian. 2000. Computer und Bildung. Eine medienanalytische Untersuchung der Computertechnologie in bildungstheoretischer Perspektive. Bielefeld: Universität Bielefeld. http://nbn-resolving.de/urn:nbn:de:hbz:361-1615.

Swertz, Christian. 2009. "Medium und Medientheorien». In Umwelten. Sozialpädagogik/Medienpädagogik/Interkulturelle und Vergleichende Erziehungswissenschaft/Umweltpädagogik, herausgegeben von Norbert Meder, Christina Allemann-Ghionda, und Uwe Uhlendorff, Handbuch der Erziehungswissenschaft Band III/2:751-80. Paderborn: Ferdinand Schöningh. 
Swertz, Christian. 2012. «Utopologische Medienpädagogik. Ein Plädoyer für das methodische Bedenken der Zukunft.» In Zukunft des Lernens. Wie digitale $\mathrm{Me-}$ dien Schule, Aus- und Weiterbildung verändern, herausgegeben von Edith Blaschitz, Gerhard Brandhofer, Christian Nosko, und Gerhard Schwed, 24. Glückstadt: Verlag Werner Hülsbusch.

Swertz, Christian. 2017a. «Rhythmuserleben mit Medien Medienpädagogische Anmerkungen zur Produktion zeitlicher Gliederungen». Medienimpulse 55 (3): 16. https://journals.univie.ac.at/index.php/mp/article/view/mill14/1260.

Swertz, Christian. 2017b. "Orientierungskönnen in der Leonardo-Welt». Erziehungswissenschaft 28 (2): 9-18. https://doi.org/10.3224/ezw.v28i2.2.

Swertz, Christian. 2020. «Bertrand Russel über Erziehung. Rekonstruktion und Dekonstruktion eines aristokratisch-liberalen Ratgeberautors.» Aufklärung und Kritik 27 (1): 198-211.

Swertz, Christian, und Katharina Mildner (Sontag). 2015. «Partizipative medienpädagogische Aktionsforschung»: Medienimpulse 53 (4). https://journals.univie.ac.at/index.php/mp/article/view/mi864.

Turing, Alan. 1936. «On Computable Numbers, with an Application to the Entscheidungsproblem». Proceedings of the London Mathematical Society 42 (2): 230-65.

Weich, Andreas. 2019. "Das „Frankfurt-Dreieck”. Ein interdisziplinäres Modell zu Bildung und Digitalisierung». Medienimpulse 57 (2): 1-19. https://doi. org/10.21243/mi-02-19-05.

Wimmer, Michael, und Alfred Schäfer. 2006. «Zwischen Fremderfahrung und Selbstauslegung». In Selbstauslegung im Anderen, 9-26. Münster: Waxmann.

Winkler, Hartmut. 2003. «Flogging a dead horse? Zum Begriff der Ideologie in der Apparatusdebatte bei Bolz und bei Kittler.» In Der kinematographische Apparat: Geschichte und Gegenwart einer interdisziplinären Debatte, herausgegeben von Robert F. Riesinger, Guntram Geser, und Lucilla Albano, 217-35. Film und Medien in der Diskussion 11. Münster: Nodus-Publ. 